Supporting Information for:

\title{
Control of the Redox Activity of Quantum Dots through Introduction of Fluoroalkanethiolates into their Ligand Shells
}

\author{
David J. Weinberg, Chen He, and Emily A. Weiss* \\ Department of Chemistry, Northwestern University, 2145 Sheridan Rd., Evanston, IL 60208 \\ *corresponding author. Email: e-weiss@ northwestern.edu
}

Synthesis and Purification of PbS QDs. Oleate-capped PbS QDs with a first excitonic peak at $985 \mathrm{~nm}$ were synthesized using a procedure adapted from that of Hines and Scholes. ${ }^{1} \mathrm{We}$ mixed $0.36 \mathrm{~g} \mathrm{PbO}$ and $2.0 \mathrm{~mL}$ oleic acid (OA) with $18.0 \mathrm{~mL}$ 1-octadecene (ODE) in a $50 \mathrm{~mL}$ three-neck round bottom flask at room temperature. Heating the mixture up to $150{ }^{\circ} \mathrm{C}$ with constant stirring under $\mathrm{N}_{2}$ flow for an hour produced a clear and colorless solution. We cooled the mixture to $110{ }^{\circ} \mathrm{C}$, and injected $0.17 \mathrm{~mL}$ of hexamethyldisilathiane dissolved in $8 \mathrm{~mL}$ of ODE. The solution turned from orange to brown within 3 seconds. After 10 minutes, we used an ice bath to cool the reaction mixture to room temperature, washed the reaction mixture with acetone ( $4: 1$ by volume), and centrifuged the mixture at $3500 \mathrm{rpm}$ for $20 \mathrm{~min}$. We decanted the supernatant, dried the QD pellet, redispersed the QDs in $10 \mathrm{~mL}$ hexanes, and precipitated the QDs two additional times, as described above, using $12.5 \mathrm{~mL}$ methanol and acetone, respectively, as the non-solvent. The cleaned PbS QDs were finally dispersed in a minimal amount of hexanes to form the stock solution.

Size Determination of $\mathrm{PbS}$ QDs via Ground State Absorption and Transmission Electron Microscopy. All ground state absorption spectra of solutions of PbS QDs were obtained on a Varian Cary 5000 spectrometer using a 1-cm quartz cuvette. We corrected the baselines of all spectra with neat solvent prior to measurement. We determined the size of the synthesized PbS QDs from the position of the first excitonic peak $(985 \mathrm{~nm})$ using the calibration curve of Moreels et al. ${ }^{2}$ All concentrations were measured from the absorbance at $400 \mathrm{~nm}$ (Figure S1A). In order to verify this size, we performed transmission electron microscopy experiments using a JEOL JEM-2100F FAST TEM. We prepared TEM samples by drop-casting a solution of PbS QDs in hexanes onto an Ultrathin Carbon on Holey Carbon Support Film (Ted Pella, Inc). Analysis of $121 \mathrm{PbS}$ QDs with the ImageJ $^{3}$ software package yields an average 
particle radius of $1.7 \pm 0.2 \mathrm{~nm}$, and the $1.6 \mathrm{~nm}$ radius predicted from the Moreels calibration curve is within the error of this measurement.
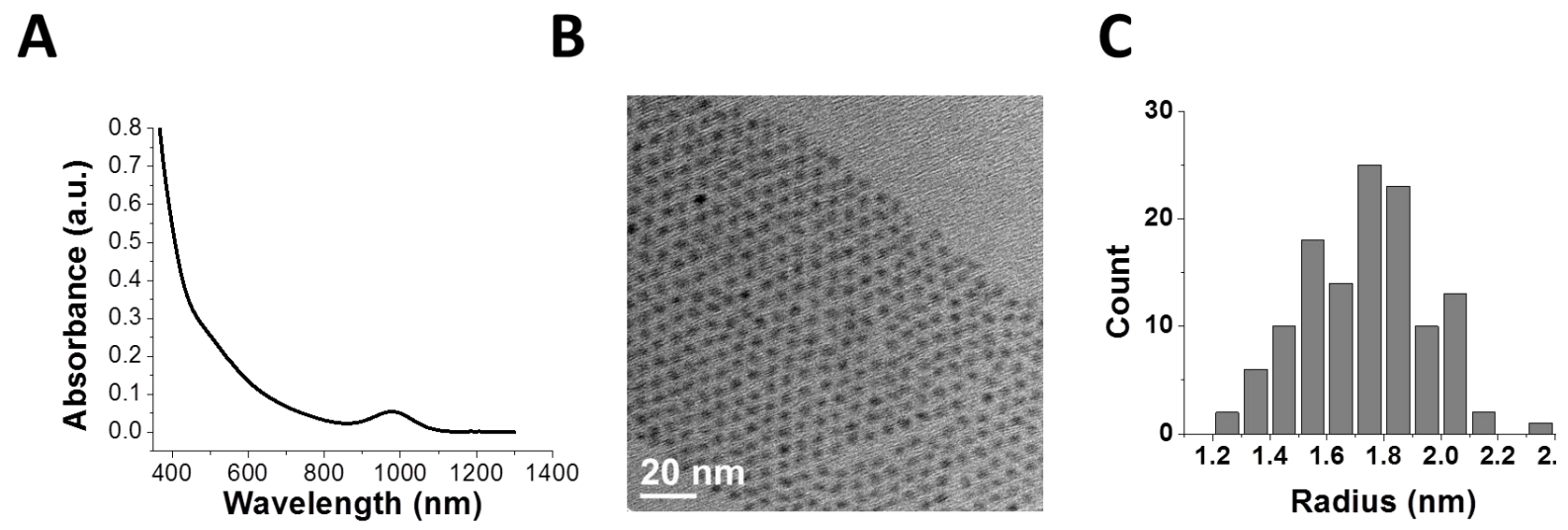

Figure S1. A) Absorbance spectrum of $0.69 \mu \mathrm{M}$ oleate-capped PbS QDs (1.6 nm radius) in hexanes. B) Representative transmission electron micrograph (TEM) image of oleate capped $\mathrm{PbS}$ QDs. C) Histogram of nanocrystal radii constructed from 121 QD diameter measurements in ImageJ. The average and standard deviation particle radius from TEM is $1.7 \pm 0.2 \mathrm{~nm}$.

\section{Quantification of the Mixed Monolayer PbS QD Ligand Shells via NMR spectroscopy.}

We performed all quantitative NMR experiments on either a Bruker Avance III $600 \mathrm{MHz}$ spectrometer $\left({ }^{1} \mathrm{H}\right.$ spectra) or an Agilent DD2 $500 \mathrm{MHz}$ system $\left({ }^{19} \mathrm{~F}\right.$ spectra).

Preparation of Samples for Quantitative ${ }^{1} H$-NMR analyses. We transferred 49 nanomoles $\mathrm{PbS}$ QDs from the stock solution (as determined from ground state absorption spectroscopy) into a scintillation vial and removed the hexanes solvent under gentle $\mathrm{N}_{2}$ flow. We brought the QD pellet into a nitrogen glovebox and resuspended the nanocrystals in $2880 \mu \mathrm{L}$ degassed $\mathrm{d}_{\sigma^{-}}$ benzene. We next dissolved $38.6 \mathrm{mg}$ biphenyl in $1 \mathrm{~mL}$ of degassed $\mathrm{d}_{\sigma}$-benzene, and added 120 $\mu \mathrm{L}$ of this biphenyl stock solution into QDs as the internal integration standard (610 eq of biphenyl per QD). We then separated the aforementioned QD stock solution into 5 scintillation vials, $500 \mu \mathrm{L}$ each, to prepare QD samples with different surface compositions individually. We added $10 \mu \mathrm{L}$ of either 1-decanethiol (DT) or $1 H, 1 H, 2 H, 2 H$-perfluorodecanethiol (PFDT) to 4 $\mathrm{mL}$ degassed $\mathrm{d}_{\sigma}$-benzene such that we prepared stock solutions of $12.0 \mathrm{mM}$ DT or $8.7 \mathrm{mM}$ PFDT. Then we added $16.6 \mu \mathrm{L}$ ( 24 eq DT) or $33.3 \mu \mathrm{L}$ (48 eq DT) of decanethiol stock solution to the QDs for the DT/OA capped samples, and we added $28.7 \mu \mathrm{L}$ (30 eq PFDT) or $57.4 \mu \mathrm{L}$ (60 eq PFDT) perfluorodecanethiol stock solution to the QDs for the PFDT/OA samples. A blank sample was prepared with no thiols added. In all cases we next added sufficient solvent to bring 
the total volume to $760 \mu \mathrm{L}$ (ie such that all solutions were $10.8 \mu \mathrm{M}$ PbS QDs in $\mathrm{d}_{6}$-benzene), and we let the samples sit under $\mathrm{N}_{2}$ for at least $3 \mathrm{~h}$ to equilibrate before we take NMR measurements.

Estimation of Bound Decanethiolate. As described in the main text, we quantify the amount of bound decanethiolate in the DT/OA mixed monolayers by determining how many DT ligands are converted into disulfides and assuming that all remaining added decanethiol binds to the surface of the PbS QDs. In order to quantify the disulfide species, we manually baseline the area surrounding the signal at $2.56 \mathrm{ppm}$ (corresponding to the disulfide protons alpha to the sulfur atoms as illustrated in Figure 1) using the MestreNova software package, then compare the integral of this peak to an internal biphenyl standard. This analysis reveals that 3 molar equivalents of decyl disulfide (DDS) are formed after the addition of 24 eq DT, and 4 molar equivalents are formed after the addition of $48 \mathrm{eq}$ DT to solutions of PbS QDs in degassed benzene. We also note that DDS added to a solution of PbS QDs neither displaces oleic acid nor exhibits broadened ${ }^{1} \mathrm{H}$ NMR peaks - that is, all decyl disulfides formed from DT on the surface of $\mathrm{PbS}$ QDs desorb from the surface.
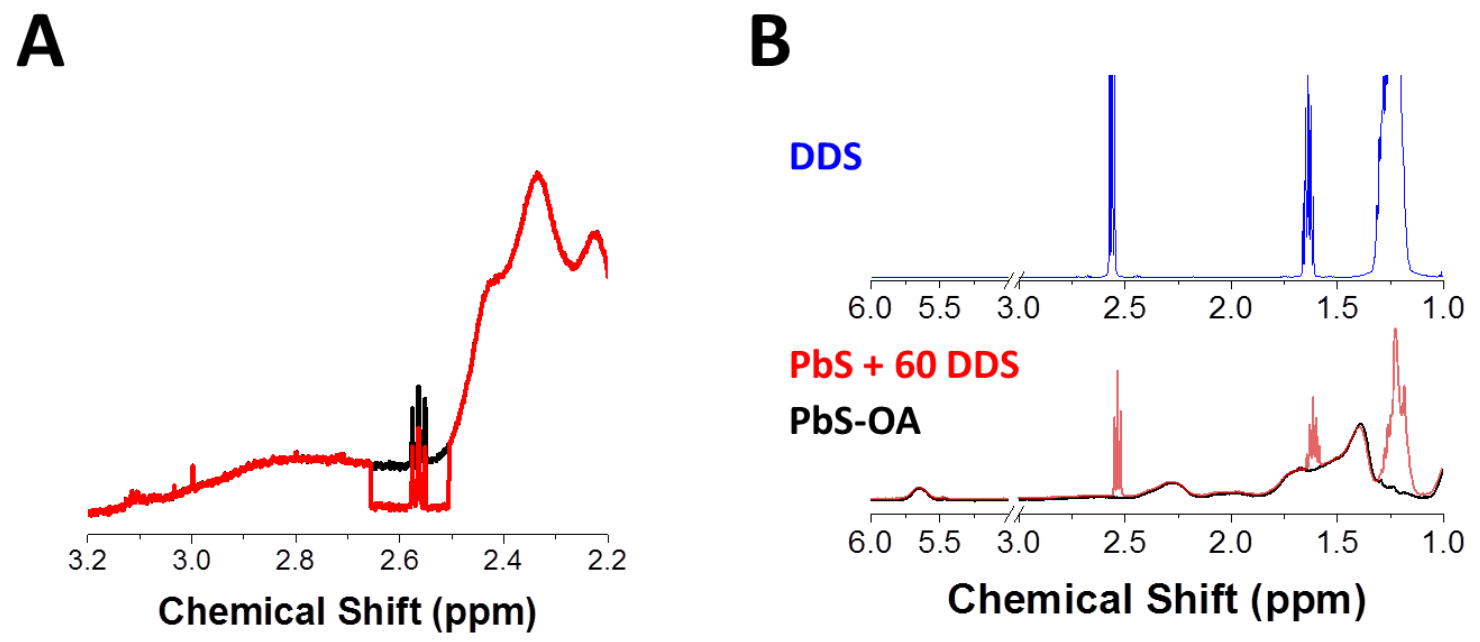

Figure S2. A) Representative ${ }^{1} \mathrm{H}$ NMR spectrum of PbS QDs treated with 48 eq DT including the decyl disulfide peak indicated in the text $(2.56 \mathrm{ppm})$ before (black) and after (red) manually baselining using MestreNova software. B) ${ }^{1} \mathrm{H}$ NMR spectrum of decyl disulfide (blue), and $\mathrm{PbS}$ QDs before (black) and after (red) treatment with 60 molar equivalents of decyl disulfide. Note that all oleate remains bound, and the decyl disulfide peaks do not broaden (indicating a lack of binding).

Determination of Bound and Free Oleate after Thiolate Treatment. We determined the total number of oleic acid molecules (bound and free) in each PbS QD sample by integrating the 
NMR signal of the two oleate vinyl protons centered at $5.69 \mathrm{ppm}$ relative to an internal biphenyl standard (7.45 ppm). The NMR peak of bound OA in the absence of thiols (Figure S3A) fits well to a single Gaussian peak, which we ascribe to homogenous broadening of the NMR lineshape due to a distribution of QD sizes and chemical environments. In order to determine the number of oleic acid ligands bound to the surface after treatment of PbS QDs with either DT or PFDT, we fit the vinyl oleate signal of these samples to a sum of two Gaussians corresponding to bound and displaced OA ligand (fitting parameters are collected in Table S1). We note that in the case of PbS QDs treated with 48 eq DT or 60 eq PFDT, all parameters of the Gaussian curves were allowed to float, and the Gaussian peak positions and widths from these fits were used to approximate the bound and free peaks for PbS QDs + 24 DT or 30 PFDT (ie parameters were manually adjusted for these samples to find the best fit). We calculate the fraction of the total signal contributed by the free and bound peaks, and multiply these fractions by the number of molar equivalents of oleic acid measured relative to the biphenyl internal standard to determine the number of bound and free OA ligands. 

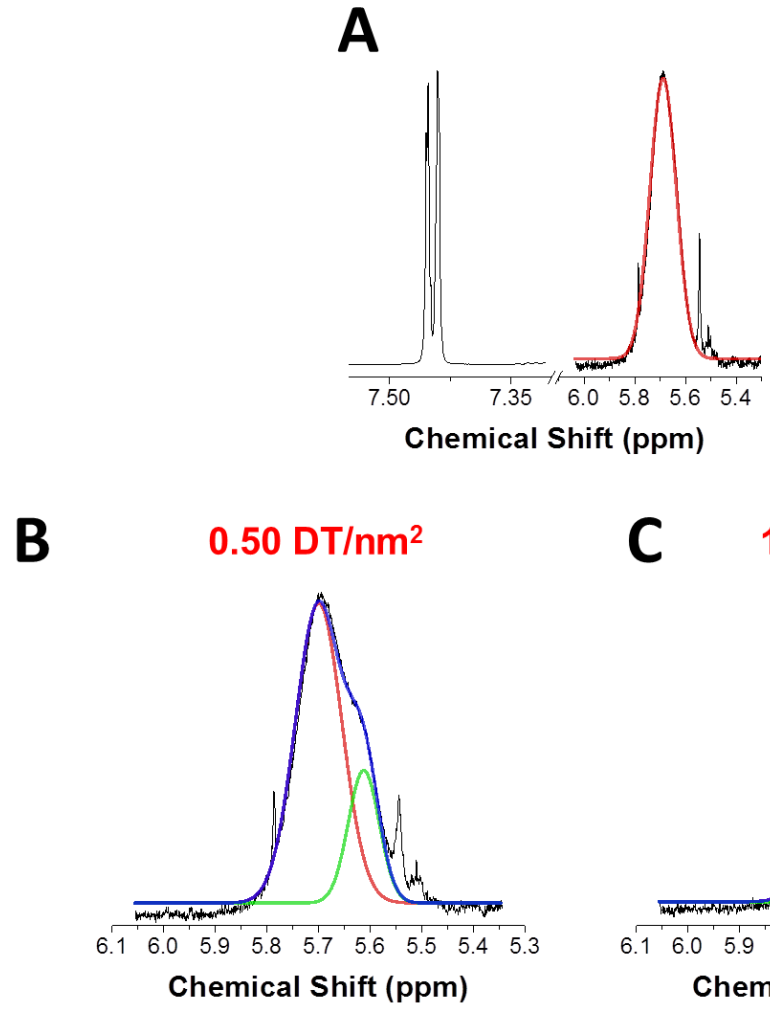

C $\quad 1.14 \mathrm{DT} / \mathrm{nm}^{2}$

D

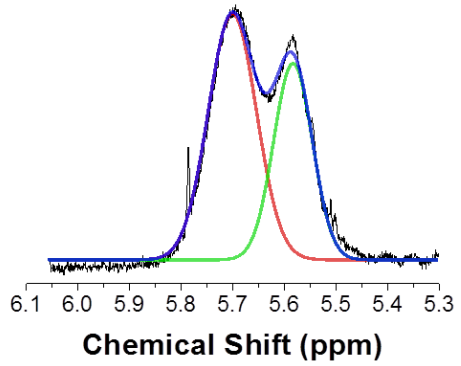

E $\quad 1.4 \mathrm{PFDT} / \mathrm{nm}^{2}$
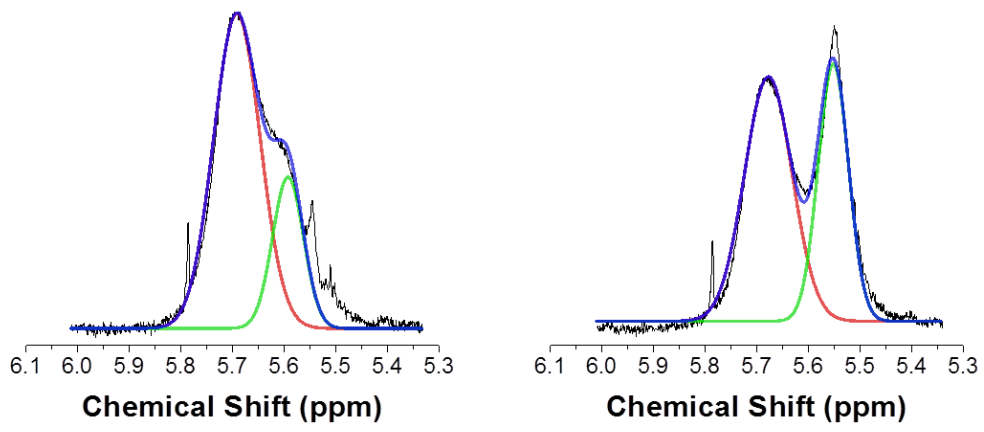

Figure S3. A) The aromatic and vinyl regions of the ${ }^{1} \mathrm{H}$ NMR spectrum of PbS QDs (10.8 $\mu \mathrm{M}$ in benzene) capped with native oleate ligand, showing both the biphenyl internal standard signal $(7.45 \mathrm{ppm})$ used to quantify bound and free oleic acid in solution, and the vinyl protons of bound oleate (5.69 ppm). B-E) The vinyl region of ${ }^{1} \mathrm{H}$ NMR spectra for PbS QDs treated with B) 24 or C) 48 molar equivalents of DT, or treated with D) 30 or E) 60 molar equivalents of PFDT. Solid lines are fits to Gaussian peaks as described in the text to distinguish the fraction of oleic acid that is bound to the surface of the quantum dot or free in solution. 
Table S1. Gaussian Fitting Parameters for the Determination of Free and Bound Oleic Acid

\begin{tabular}{|l|l|l|l|l|l|l|}
\hline & \multicolumn{3}{|c|}{ Peak 1 (bound OA) } & \multicolumn{3}{c|}{ Peak 2 (displaced OA) } \\
\hline & $\begin{array}{c}\text { Center } \\
(\mathbf{p p m})\end{array}$ & $\begin{array}{c}\text { Width } \\
(\mathbf{p p m})\end{array}$ & Area & $\begin{array}{c}\text { Center } \\
(\mathbf{p p m})\end{array}$ & $\begin{array}{c}\text { Width } \\
(\mathbf{p p m})\end{array}$ & Area \\
\hline PbS OA & 5.68 & 0.1 & 57.0 & - & - & - \\
\hline $\begin{array}{l}\text { PbS + 30 } \\
\text { PFDT }\end{array}$ & 5.69 & 0.09 & 45.0 & 5.59 & 0.06 & 14.4 \\
\hline $\begin{array}{l}\text { PbS + 60 } \\
\text { PFDT }\end{array}$ & 5.68 & 0.09 & 35.7 & 5.54 & 0.06 & 24.1 \\
\hline PbS + 24 DT & 5.70 & 0.09 & 42.7 & 5.61 & 0.06 & 12.6 \\
\hline PbS + 48 DT & 5.70 & 0.09 & 36.4 & 5.58 & 0.075 & 23.3 \\
\hline
\end{tabular}

Quantification of Bound PFDT via ${ }^{19}$ F NMR. We first verified that the integrated intensity of the PFDT terminal $\mathrm{CF}_{3}$ signal described in the main text does not change relative to an internal 2-fluorobiphenyl standard in the presence $\mathrm{PbS}$ QDs (i.e., the integrated intensity of the $\mathrm{CF}_{3}$ signal does not decrease upon binding to the QD), Figure S4A. We then measured the ${ }^{19} \mathrm{~F}$ NMR spectra of PbS QDs treated with 30 or 60 eq PFDT, Figure S4B,C, and fit this $\mathrm{CF}_{3}$ signal as a sum of two Lorentzian functions (whose fitting parameters are summarized in Table S2), in which the narrow signal corresponds to free thiol and the broadened peak corresponds to fluorinethiols bound to the QD surface. We calculate the fraction of the total signal contributed by the free and bound Lorentzian peaks, and multiply these fractions by the number of molar equivalents PFDT added to the $\mathrm{PbS}$ QDs to determine the number of bound and free PFDT ligands. We observed no sample precipitation at these PFDT coverages, therefore all added PFDT is either free or bound to the surface.

Table S2. Lorentzian Fitting Parameters for the Determination of Free and Bound PFDT.

\begin{tabular}{|l|l|l|l|l|l|l|}
\hline & \multicolumn{3}{|c|}{ Peak 1 (bound PFDT) } & \multicolumn{3}{c|}{ Peak 2 (free PFDT) } \\
\hline & $\begin{array}{c}\text { Center } \\
(\mathbf{p p m})\end{array}$ & $\begin{array}{c}\text { Width } \\
(\mathbf{p p m})\end{array}$ & Area & $\begin{array}{c}\text { Center } \\
(\mathbf{p p m})\end{array}$ & $\begin{array}{c}\text { Width } \\
(\mathbf{p p m})\end{array}$ & Area \\
\hline $\begin{array}{l}\text { PbS + 30 } \\
\text { PFDT }\end{array}$ & -81.3 & 0.44 & 606 & -81.1 & 0.026 & 214 \\
\hline $\begin{array}{l}\text { PbS + 60 } \\
\text { PFDT }\end{array}$ & -81.4 & 0.45 & 1511 & -81.1 & 0.028 & 231 \\
\hline
\end{tabular}



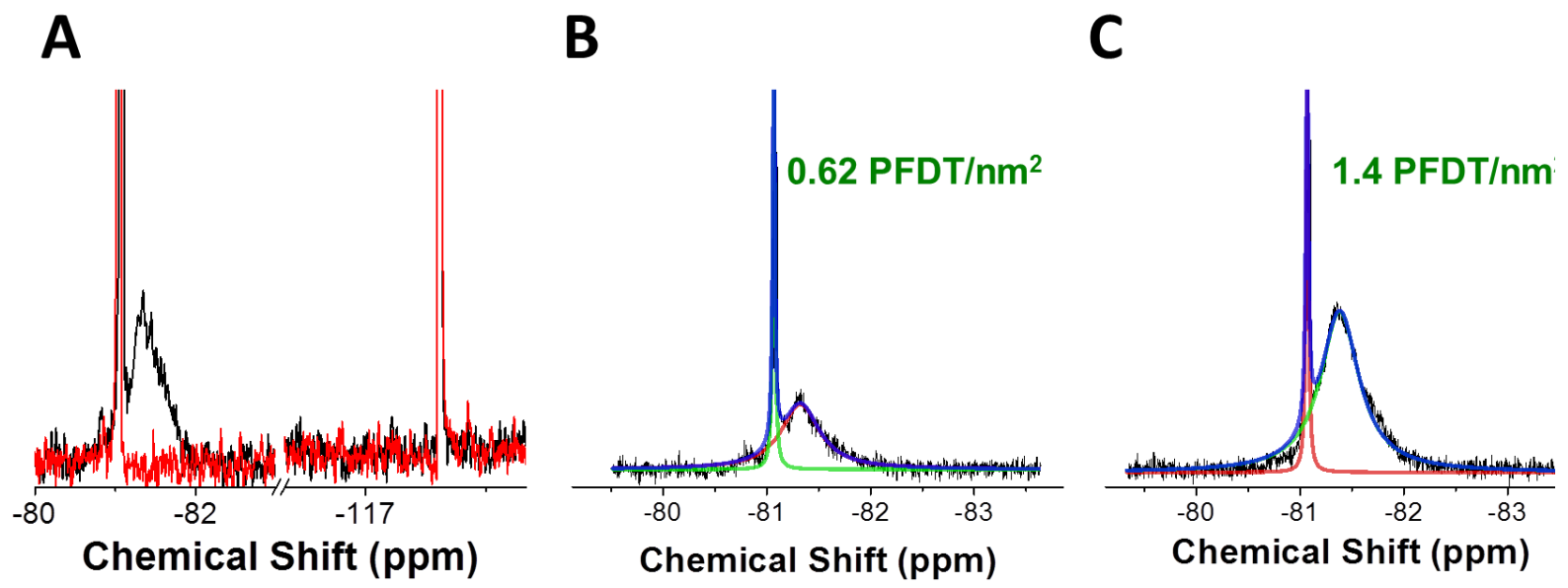

Figure S4. A) The terminal $\mathrm{CF}_{3}$ fluorine signal (-81.3 ppm) of PFDT before (red) and after (black) the addition of 0.021 eq PbS QDs (such that there are 49 molar equivalents of PFDT per QD). Integration of this signal relative to the $1.6 \mathrm{mM} 2$-fluorobiphenyl internal standard ($117.9 \mathrm{ppm})$ reveals that the measured concentration of PFDT does not change within 5\% in the presence of QDs $\left(0.53 \mathrm{mM}\right.$ versus $0.56 \mathrm{mM}$ PFDT). B,C) The terminal $\mathrm{CF}_{3}$ region of ${ }^{19} \mathrm{~F}$ NMR spectra for PbS QDs treated with B) 30 or C) 60 molar equivalents of PFDT. Solid lines are fits to Lorentzian peaks as described in the text to distinguish the fraction of PFDT that is bound to the surface of the quantum dot or free in solution.

Determination of NMR Quantification Error via Calibration Curves. In order to determine the error in NMR measurements of ligand coverage, we prepared calibration samples with known concentrations of PFDT, DDS, or OA, and then measured the concentration of these samples relative an internal 2-fluorobiphenyl (PFDT) or unsubstituted biphenyl (DDS, OA) standard. We plot the measured versus added concentration for each molecule, and fit each of these plots to a line with a slope of unity which intercepts at the origin (i.e., the ideal 1:1 response line, Figure S5). We estimate the error in our NMR measurements as the average percent residual between the fit and measured data. The decyl disulfide residuals are on average $10 \%$ of the measured values, the oleic acid measurements yield an $11 \%$ error, while the PFDT curve has a $6 \%$ residual error. These fractional errors are multiplied by the measured number of molar equivalents of ligand to determine the error in ligand count (ie 220 OA/QD has an error of 20). We note that the ${ }^{19} \mathrm{~F}$ NMR spectra for the PFDT calibration plot were collected using a relaxation delay of $15 \mathrm{~s}$ while all other ${ }^{19} \mathrm{~F}$ measurements were collected with a delay of $1 \mathrm{~s}$, but that the measured fraction of bound vs free PFDT ligand does not differ when measured at these two relaxation delay times. 

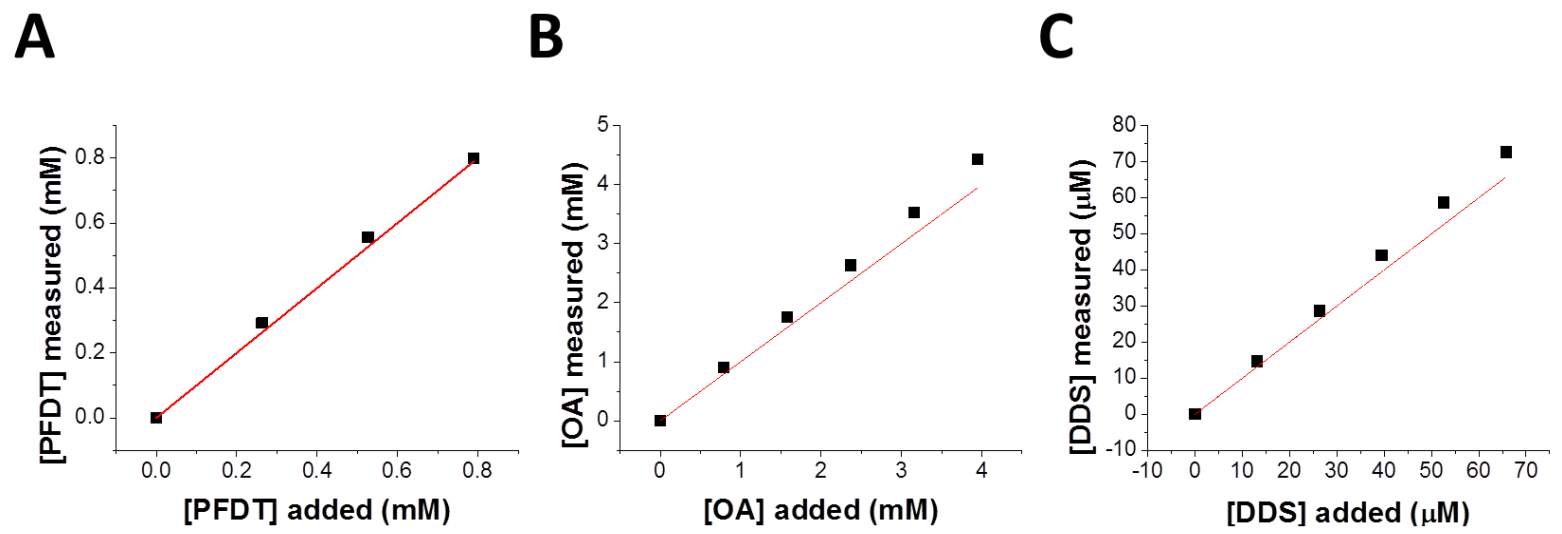

Figure S5. A) Plot of the measured concentration of PFDT versus concentration added as determined by comparing the terminal $\mathrm{CF}_{3}$ signal to an internal 2-fluorobiphenyl standard. Similar plots were constructed for B) oleic acid (measured at $5.61 \mathrm{ppm}$ ) and C) decyl disulfide (DDS, $2.56 \mathrm{ppm}$ ) measured relative to an internal biphenyl standard. All measurements were taken in the absence of $\mathrm{PbS}$ QDs. Solid lines indicate fits to a line of slope $=1$, intercept $=0$, and the deviation from this ideal fit are used to calculate errors in measured concentration as described in the text.

\section{Photoluminescence Spectroscopy of Thiol-treated PbS QD Samples after the Addition} of s-BQs. Preparation of Thiol-treated PbS QDs for Photoluminescence Experiments. We transferred 58 nanomoles $\mathrm{PbS}$ QDs from the stock solution (as determined from ground state absorption spectroscopy) into a scintillation vial and removed the hexanes solvent under gentle $\mathrm{N}_{2}$ flow. We brought the QD solid into a nitrogen glovebox and resuspended them in $4800 \mu \mathrm{L}$ degassed benzene. We next added $10 \mu \mathrm{L}$ of either 1 -decanethiol (DT) or $1 H, 1 H, 2 H, 2 H$ perfluorodecanethiol (PFDT) to $1 \mathrm{~mL}$ degassed benzene such that we prepared stock solutions of $34.6 \mathrm{mM}$ PFDT or $47.8 \mathrm{mM}$ DT. We added $29.3 \mu \mathrm{L}$ (24 eq DT) or $58.6 \mu \mathrm{L}$ (48 eq DT) of decanethiol stock solution to the quantum dots for the DT/OA capped samples, and we added 50 $\mu \mathrm{L}$ (30 eq PFDT) or $100 \mu \mathrm{L}$ (60 eq PFDT) perfluorodecanethiol stock solution to the QDs for the PFDT/OA samples. In all cases we next added sufficient solvent to bring the total volume to $4900 \mu \mathrm{L}$ (ie such that all solutions were 11.5 $\mu \mathrm{M}$ PbS QDs in benzene), and the solutions were allowed to sit under $\mathrm{N}_{2}$ for at least $3 \mathrm{~h}$.

Preparation of PbS-sBQ Quenching Samples. We prepared a $1 \mathrm{~mL}$ stock solution of $0.3 \mathrm{M}$ $\mathrm{Me}_{2} \mathrm{BQ}$ or $\mathrm{Me}_{4} \mathrm{BQ}$ in degassed benzene. We next separated the batch of thiol treated QDs into 5 vials each with $700 \mu \mathrm{L}$ QD-thiol stock solution. Finally, we added 0, 15, 30, 45, or $60 \mu \mathrm{L}$ benzoquinone stock solution to each aliquot $(0,548,1095,1643$, of 2190 molar equivalents s- 
$\mathrm{BQ})$, and $60,45,30,15$, or $0 \mu \mathrm{L}$ degassed benzene to each sample to ensure constant volume. Samples were allowed to equilibrate for at least $12 \mathrm{~h}$, and used as is for PL or transient absorption measurements.

Spectra of Thiol-treated PbS QDs Before and After Addition of DT, PFDT, and s-BQ. Photoluminescence spectra were collected on a Horiba Fluorolog-3 spectrofluorimeter in the right-angle geometry using a $2 \mathrm{~mm} / 10 \mathrm{~mm}$ dual path-length cuvette (in which we excite along the $10 \mathrm{~mm}$ axis of the cuvette). All solutions were photoexcited at $800 \mathrm{~nm}$, and we divided the measured intensity by the instantaneous intensity of the excitation source.

We observe a bathochromic ("red") shift in the QD band edge absorption of the QDs upon the addition of DT and PFDT (Figure S6), which is consistent with the delocalizing nature of bound thiolate species. ${ }^{4}$ This bathochromic shift clearly dominates any hypsochromic shift that would accompany loss of lead oleate species from the QD surface upon addition of thiols, if the lead atoms were part of the QD core. Treatment of the QDs with these thiols does not decrease the relative quantum yield of the nanocrystals when normalized by absorbance at the excitation wavelength (and in fact increases the relative quantum yield in the case of PFDT, Figure S6), which is consistent with passivation of the QD surface rather than etching of the PbS QD core. ${ }^{5}$
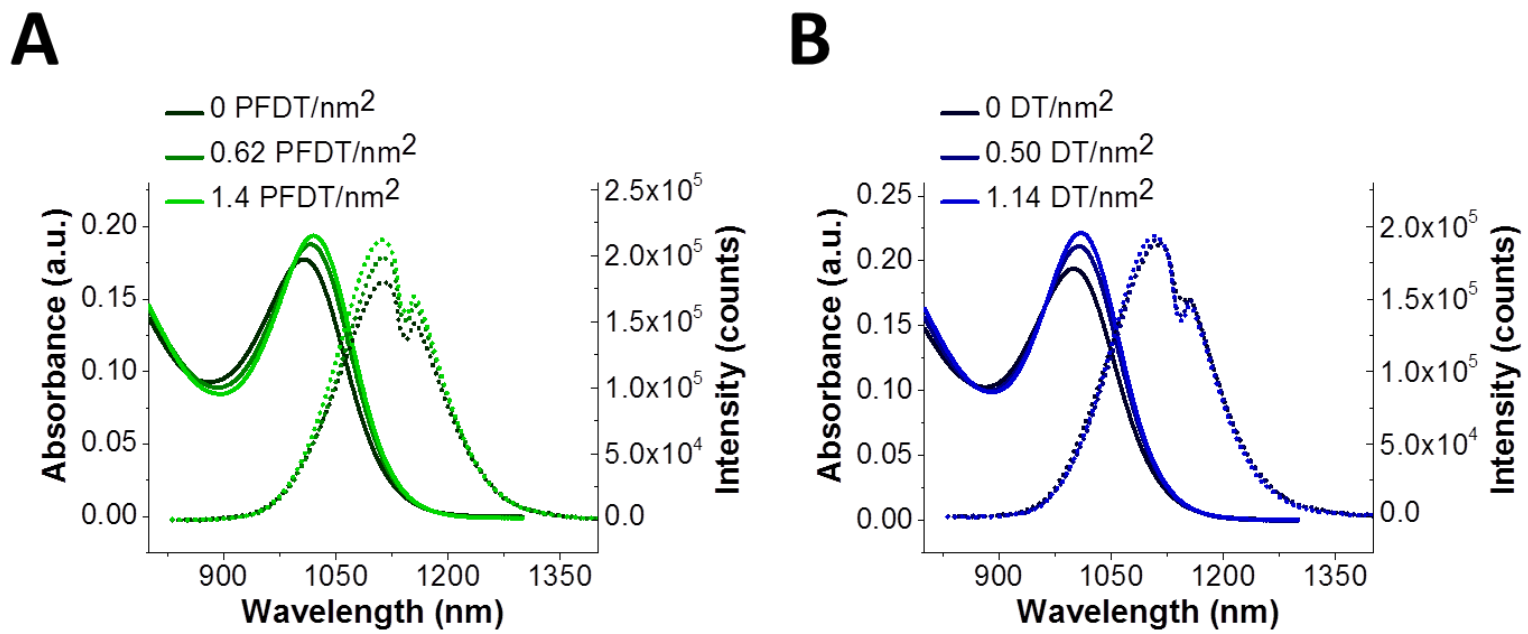

Figure S6. Absorbance (solid lines) and photoluminescence (dotted lines) spectra of $\mathrm{PbS}$ QDs $\left(11.5 \mu \mathrm{M}\right.$ in benzene) coated with A) $0,0.62$, or $1.4 \mathrm{PFDT} / \mathrm{nm}^{2}$ or B) $0,0.5,1.14$ $\mathrm{DT} / \mathrm{nm}^{2}$. All photoluminescence spectra were collected following photoexcitation at $800 \mathrm{~nm}$ and are normalized to the absorbance at that wavelength, and the "dip" at $1120 \mathrm{~nm}$ is attenuation of signal by absorption of a vibrational overtone of the solvent. This incomplete subtraction of solvent signal obscures any red shift in PL peak that accompanies the red-shift in absorption. 

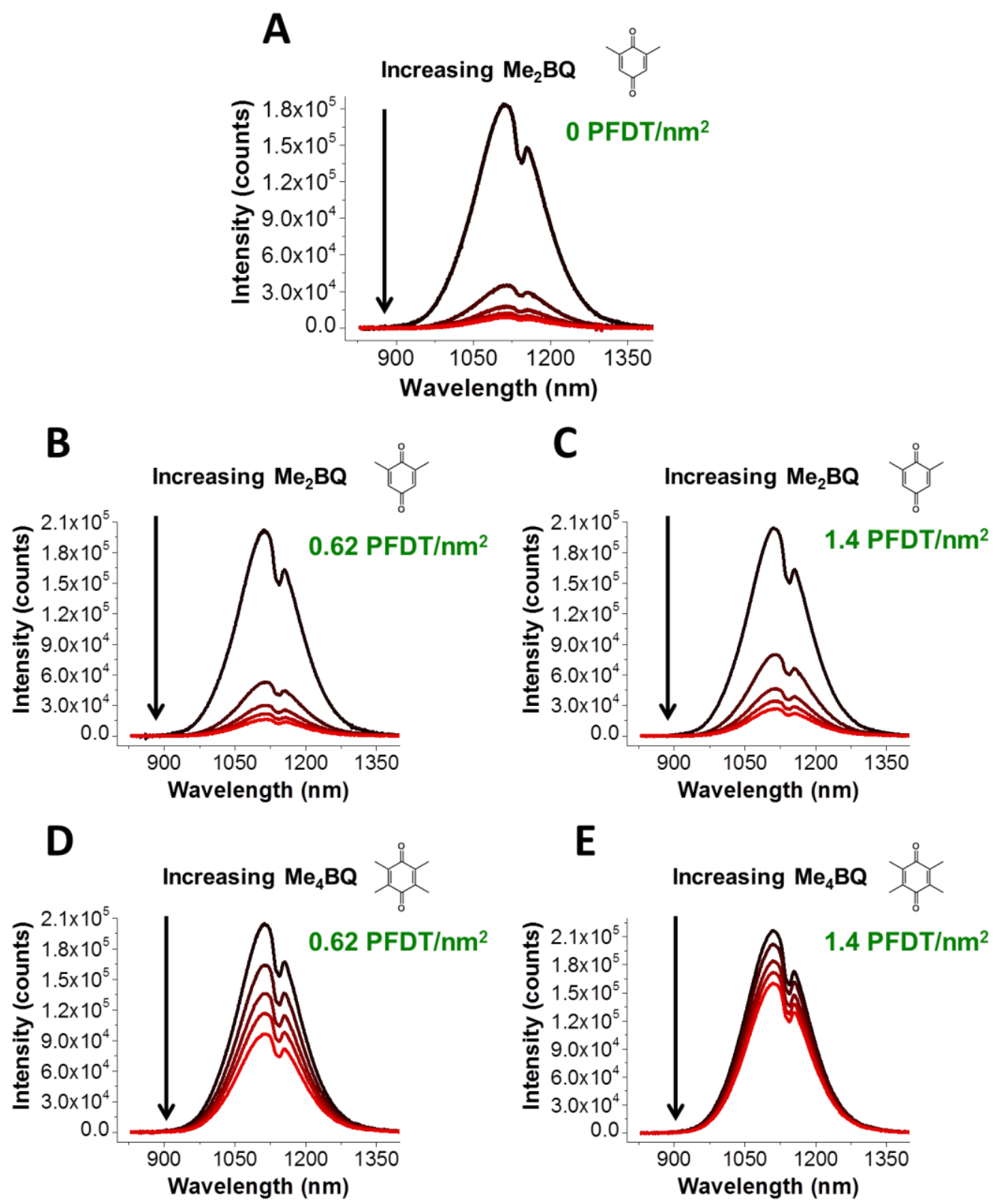

Figure S7. Photoluminescence spectra of PbS QDs (11.5 $\mu \mathrm{M}$ in benzene) coated with A) 0 PFDT $/ \mathrm{nm}^{2}$ (i.e. OA capped PbS QDs), B) $0.62 \mathrm{PFDT} / \mathrm{nm}^{2}$ or C) $1.4 \mathrm{PFDT} / \mathrm{nm}^{2}$ after the addition of 0-2190 molar equivalents of $\mathrm{Me}_{2} \mathrm{BQ}$. D,E) Photoluminescence spectra of the samples described in B),C) after the addition of $\mathrm{Me}_{4} \mathrm{BQ}$ following photoexcitation at $800 \mathrm{~nm}$. The "dip" at $1120 \mathrm{~nm}$ is attenuation due to absorption of a vibrational overtone of the solvent. 

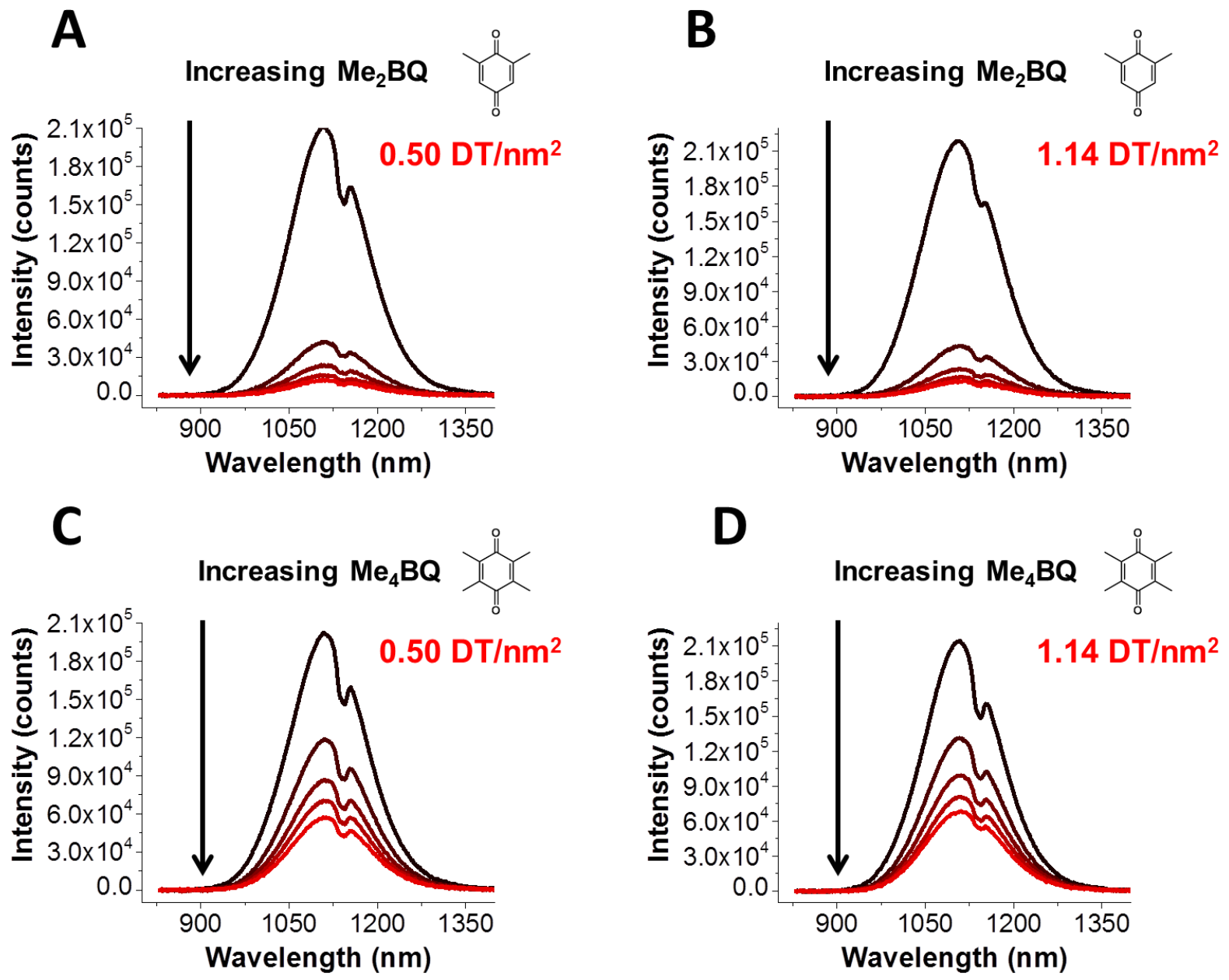

Figure S8. Photoluminescence spectra of PbS QDs (11.5 $\mu \mathrm{M}$ in benzene) coated with A) 0.50 $\mathrm{DT} / \mathrm{nm}^{2}$ or B) $1.14 \mathrm{DT} / \mathrm{nm}^{2}$ after the addition of 0-2190 molar equivalents of $\mathrm{Me}_{4} \mathrm{BQ}$. C,D) Photoluminescence spectra of the samples described in $\mathbf{A}$ ),B) after the addition of $\mathrm{Me}_{2} \mathrm{BQ}$ following photoexcitation at $800 \mathrm{~nm}$. The "dip" at $1120 \mathrm{~nm}$ is attenuation due to absorption of a vibrational overtone of the solvent.

\section{Transient Absorption Spectroscopy of Thiol-treated PbS Samples after the Addition of} s-BQs. Transient Absorption Apparatus. Our picosecond-nanosecond and nanosecond microsecond transient absorption spectrometers are described in detail elsewhere. ${ }^{6,7}$ All transient absorption measurements were made in $2 \mathrm{~mm}$ glass cuvettes while stirring with a Teflon stir bar. In order to avoid the contribution of multiple exciton dynamics, the expectation value of excitons per QD was adjusted to be less than or equal to $<0.3>$ for all ultrafast experiments.

Microsecond Transient Absorption. We monitored the dynamics of ground state bleach recovery on the nanosecond-microsecond timescale for PbS QDs capped with 0, 0.62 or 1.4 
PFDT/nm² following the addition of 0-2190 molar equivalents of $\mathrm{Me}_{2} \mathrm{BQ}$ or $\mathrm{Me}_{4} \mathrm{BQ}$, Figure 2 and Figure S9 With the exception of the PbS QDs capped with $1.4 \mathrm{PFDT} / \mathrm{nm}^{2}$ and treated with $\mathrm{Me}_{2} \mathrm{BQ}$, each of these traces fits well to a monoexponential decay convoluted with a Gaussian instrument response function, eq $\mathrm{S} 1$.

$$
\Delta O D=I R F \cdot A_{1} e^{-t / \tau}
$$

The PbS QDs capped with 1.4 PFDT/ $\mathrm{nm}^{2}$ and treated with 0-2190 molar equivalents of $\mathrm{Me}_{2} \mathrm{BQ}$ were fit as a sum of two exponential decays convoluted with an instrument response function, which is, in this case, a Gaussian pulse (eq S2).

$$
y=I R F \otimes\left[A_{1} e^{\frac{-\left(t-t_{0}\right)}{\tau_{1}}}+A_{2} e^{\frac{-\left(t-t_{0}\right)}{\tau_{2}}}\right]
$$

These traces require an additional component on the tens of ns timescale comprising $\sim 15 \%$ of the signal intensity, which is consistent with a "long" time ascribed to surface mediating trapping observed in ultrafast transient absorption experiments (see Figures S11 and S12). This slight inconsistency is not surprising given the variability of QD samples, and adding an additional exponential does not significantly change the measured time constant assigned to excitonic decay. The results of these fits are summarized in Table S3. 


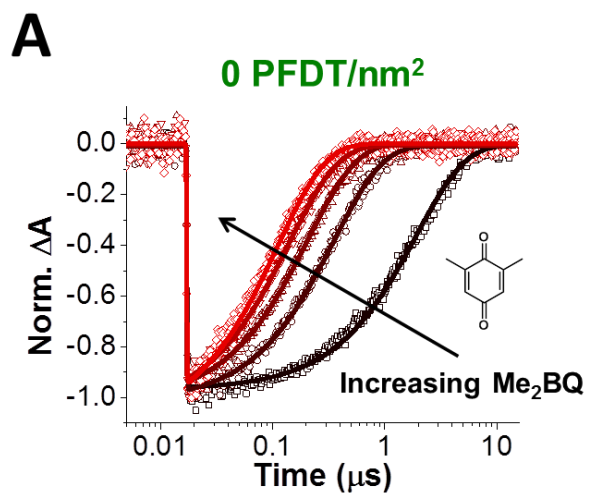

B

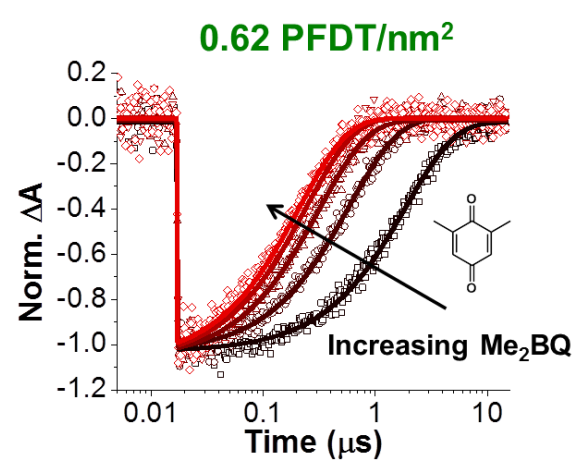

D

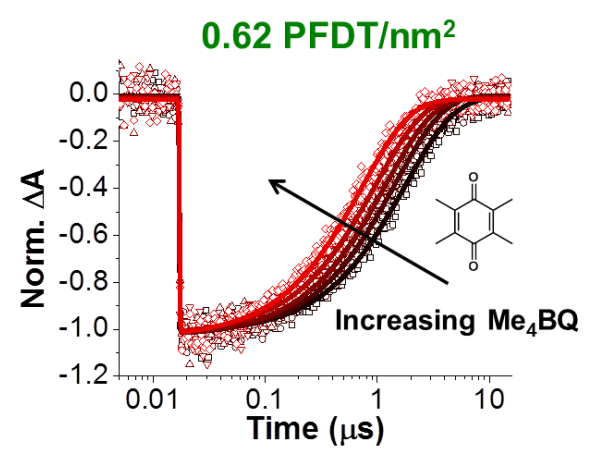

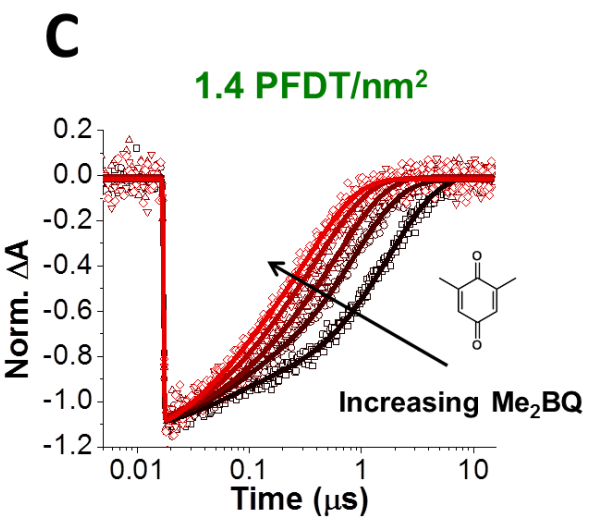

E

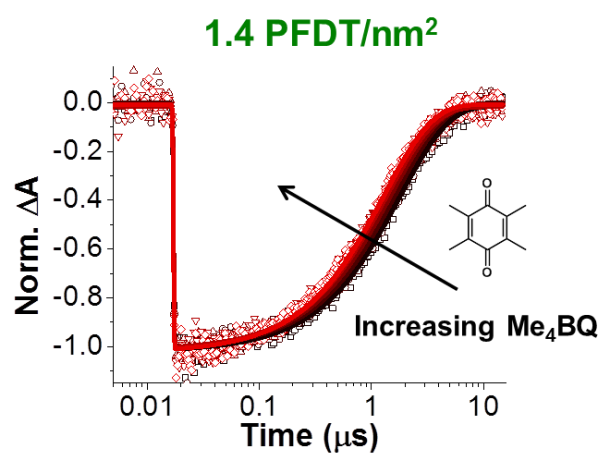

Figure S9. Transient kinetic traces of $\mathrm{PbS}$ QDs $(11.5 \mu \mathrm{M}$ in benzene) coated with A) 0 PFDT $/ \mathrm{nm}^{2}$ (i.e., OA capped PbS QDs, extracted at $990 \mathrm{~nm}$ ), B) 0.62 PFDT (extracted at 995 $\mathrm{nm}$ ) or C) 1.4 PFDT (extracted at $1005 \mathrm{~nm}$ ) after the addition of 0-2190 molar equivalents of $\mathrm{Me}_{2} \mathrm{BQ}$ following photoexcitation at $850 \mathrm{~nm}$. D,E) Transient kinetic traces of the samples described in B),C) after the addition of 0-2190 molar equivalents of $\mathrm{Me}_{4} \mathrm{BQ}$ following photoexcitation at $850 \mathrm{~nm}$. Solid lines are fits to equation $\mathrm{S} 1$ or $\mathrm{S} 2$ as described in the text. 
Table S3. Fitting parameters for $\mathrm{PbS}$ QDs coated with $0,0.62$, or $1.4 \mathrm{PFDT} / \mathrm{nm}^{2}$ after the addition of s-BQ quencher.

\begin{tabular}{|c|c|c|c|c|c|c|c|}
\hline & \multicolumn{4}{|c|}{$\mathrm{Me}_{2} \mathrm{BQ}$} & \multicolumn{3}{|c|}{$\mathrm{Me}_{4} \mathrm{BQ}$} \\
\hline \multirow[t]{2}{*}{$\begin{array}{c}\text { eq } \\
\text { s-BQ }\end{array}$} & $\begin{array}{c}0 \\
\text { PFDT/nm }\end{array}$ & $\begin{array}{c}0.62 \\
\text { PFDT/nm } \\
\end{array}$ & \multicolumn{2}{|c|}{$\begin{array}{c}1.4 \\
\text { PFDT/nm } \\
\end{array}$} & $\begin{array}{c}0 \\
\text { PFDT/nm }\end{array}$ & $\begin{array}{c}0.62 \\
\text { PFDT/nm } \\
\end{array}$ & $\begin{array}{c}1.4 \\
\text { PFDT/nm }\end{array}$ \\
\hline & $\tau_{1}(\mu s)$ & $\tau_{1}(\mu s)$ & $\begin{array}{c}\tau_{1}(\mathrm{~ns}) \\
\left(\mathbf{A}_{1}\right)\end{array}$ & $\begin{array}{c}\tau_{2}(\mu s) \\
\left(\mathbf{A}_{2}\right)\end{array}$ & $\tau_{1}(\mu s)$ & $\tau_{1}(\mu s)$ & $\tau_{1}(\mu \mathrm{s})$ \\
\hline 0 & $1.92 \pm 0.02$ & $1.88 \pm 0.02$ & $\begin{array}{l}51 \pm 7 \\
(-0.13)\end{array}$ & $\begin{array}{l}1.78 \pm 0.02 \\
(-0.87)\end{array}$ & $2.00 \pm 0.03$ & $1.79 \pm 0.02$ & $1.80 \pm 0.02$ \\
\hline 548 & $0.354 \pm 0.003$ & $0.59 \pm 0.01$ & $\begin{array}{l}60 \pm 10 \\
(-0.15)\end{array}$ & $\begin{array}{l}0.86 \pm 0.02 \\
(-0.85)\end{array}$ & $1.04 \pm 0.02$ & $1.44 \pm 0.02$ & $1.66 \pm 0.02$ \\
\hline 1095 & $0.208 \pm 0.002$ & $0.32 \pm 0.01$ & $\begin{array}{l}80 \pm 10 \\
(-0.17)\end{array}$ & $\begin{array}{l}0.64 \pm 0.02 \\
(-0.83)\end{array}$ & $0.74 \pm 0.02$ & $1.18 \pm 0.02$ & $1.50 \pm 0.02$ \\
\hline 1643 & $0.14 \pm 0.01$ & $0.241 \pm 0.004$ & $\begin{array}{l}70 \pm 20 \\
(-0.20) \\
\end{array}$ & $\begin{array}{l}0.45 \pm 0.02 \\
(-0.80)\end{array}$ & $0.56 \pm 0.01$ & $0.97 \pm 0.01$ & $1.36 \pm 0.01$ \\
\hline 2190 & $0.11 \pm 0.001$ & $0.21 \pm 0.003$ & $\begin{array}{l}50 \pm 7 \\
(-0.21) \\
\end{array}$ & $\begin{array}{l}0.35 \pm 0.01 \\
(-0.79) \\
\end{array}$ & $0.44 \pm 0.01$ & $0.73 \pm 0.01$ & $1.30 \pm 0.01$ \\
\hline
\end{tabular}

We also measured the lifetime of $\mathrm{PbS}$ QDs treated with 24 or 48 molar equivalents of decanethiol, Figure S10, and both measured lifetimes are $1.69 \pm 0.02 \mu \mathrm{s}$.

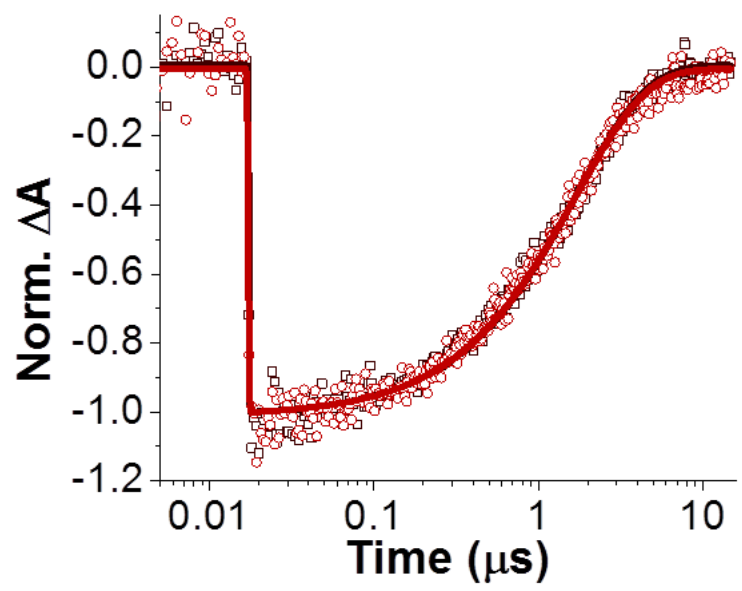

Figure S10. Transient kinetic traces of $\mathrm{PbS}$ QDs $(11.5 \mu \mathrm{M}$ in benzene) coated with 0.50 $\mathrm{DT} / \mathrm{nm}^{2}$ (extracted at $1000 \mathrm{~nm}$, black) or $1.14 \mathrm{DT} / \mathrm{nm}^{2}$ (extracted at $1007 \mathrm{~nm}$, red) following photoexcitation at $850 \mathrm{~nm}$.

Picosecond Transient Absorption. In order to determine the extent to which pre-adsorbed sBQs participate in static quenching with $\mathrm{PbS}$ QDs, we conducted femtosecond-picosecond timescale transient absorption experiments. We photoexcite the samples used for photoluminescence experiments at $850 \mathrm{~nm}$, and extract kinetics at the peak of the ground state bleach (Figure S11). We first conducted a series of TA experiments on PbS QDs coated with 0, 
0.62, or $1.4 \mathrm{PFDT} / \mathrm{nm}^{2}$ following the addition of 0 , 1095, or 2190 molar equivalents of tetramethyl benzoquinone.

We note that in the absence of both s-BQ quencher and fluorinated thiol (i.e., as-synthesized, oleate capped $\mathrm{PbS} \mathrm{QDs}$ ), the ground state recovery dynamics are not flat on the picosecond timescale. The ground state bleach recovery can be fit as a sum of two exponential decays convoluted with an instrument response function, which is, in this case, a Gaussian pulse (eq S2).

The normalized signal includes a 0.8 ps component $(6 \%)$ which is attributable to a small contribution of Auger recombination, and a 22 ns time constant (94\%) which is likely some trapping process that is intrinsic to this PbS QD synthesis. We also note that the addition of 1095 or 2190 molar equivalents of $\mathrm{Me}_{4} \mathrm{BQ}$ quencher does not change the picosecond timescale dynamics of $\mathrm{PbS}$ QDs coated with $0,0.62$, or $1.4 \mathrm{PFDT} / \mathrm{nm}^{2}$ within the signal to noise of the experiment, which provides direct evidence for a lack of static electron transfer.
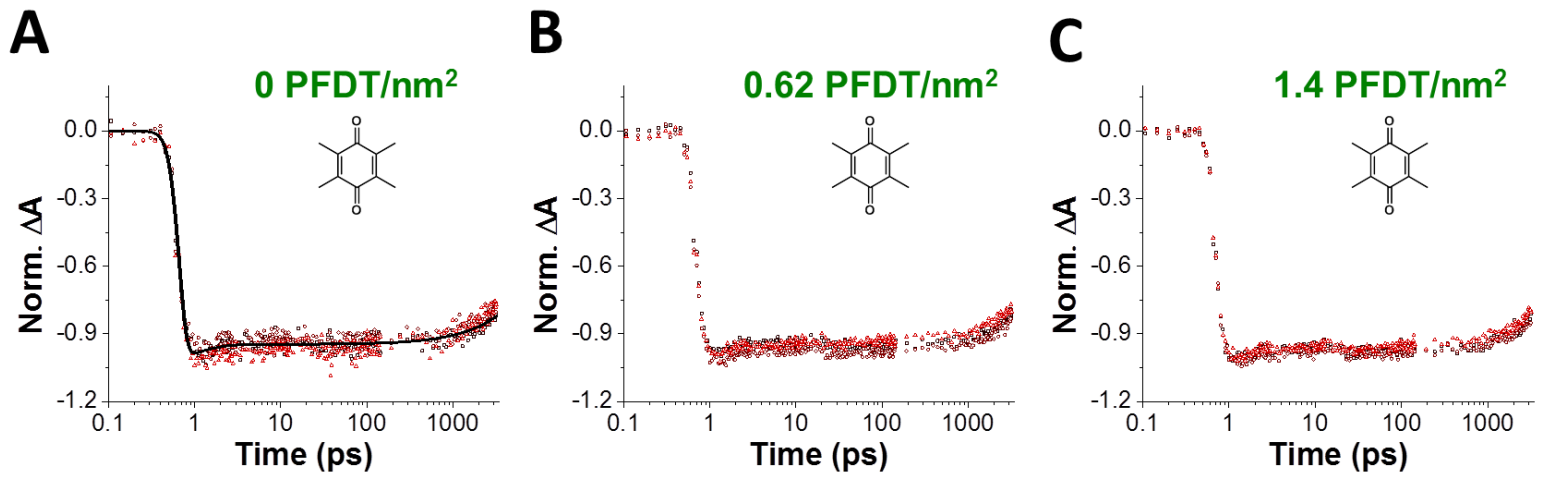

Figure S11. Transient kinetics of PbS QDs capped with A) 0, B) 0.62, or C) $1.4 \mathrm{PFDT} / \mathrm{nm}^{2}$ extracted at $983 \mathrm{~nm}, 990 \mathrm{~nm}$, and $995 \mathrm{~nm}$ respectively following photoexcitation at $850 \mathrm{~nm}$. There is no measurable change in picosecond timescale dynamics before (black) and after the addition of 1095 (maroon), or 2190 (red) molar equivalents of $\mathrm{Me}_{4} \mathrm{BQ}$. The solid black line in A) is a fit described in the text.

We conducted a similar set of picosecond transient absorption experiments for PbS QDs coated with 0, 0.62, or 1.4 PFDT/nm² treated with 0-2190 molar equivalents of $\mathrm{Me}_{2} \mathrm{BQ}$, Figure S12. For the PFDT/OA capped QDs, we find that the addition of $\mathrm{Me}_{2} \mathrm{BQ}$ does not accelerate the recovery of the QD ground state bleach. However, in the case of $\mathrm{PbS}$ capped with native oleate ligand, we find that the addition of $\mathrm{Me}_{2} \mathrm{BQ}$ introduces an additional exponential component into 
the recovery of the bleach, which must be fitted with three exponentials rather than two (see Table S4).

$$
y=I R F \otimes\left[A_{1} e^{\frac{-\left(t-t_{0}\right)}{\tau_{1}}}+A_{2} e^{\frac{-\left(t-t_{0}\right)}{\tau_{2}}}+A_{3} e^{\frac{-\left(t-t_{0}\right)}{\tau_{3}}}\right]
$$

Table S4. Fitting Parameters for Picosecond-Timescale Charge Transfer in Oleate-Capped PbS QDs to $\mathrm{Me}_{2} \mathrm{BQ}$

\begin{tabular}{|c|l|l|l|}
\hline $\mathbf{E q} \mathbf{M e}_{2} \mathbf{B Q}$ & $\begin{array}{l}\boldsymbol{\tau}_{\mathbf{1}}(\mathbf{p s}) \\
\left(\mathbf{A}_{\mathbf{1}}\right)\end{array}$ & $\begin{array}{l}\boldsymbol{\tau}_{\mathbf{2}} \mathbf{( p s )} \\
\left(\mathbf{A}_{\mathbf{2}}\right)\end{array}$ & $\begin{array}{l}\boldsymbol{\tau}_{\mathbf{3}}(\mathbf{n s}) \\
\left(\mathbf{A}_{\mathbf{3}}\right)\end{array}$ \\
\hline 0 & $1.6 \pm 0.2$ & - & 19 \\
$(-0.05)$ & & $(-0.95)$ \\
\hline 548 & $1.6 \pm 0.2$ & - & 19 \\
& $(-0.05)$ & & $(-0.95)$ \\
\hline 1095 & $1.6 \pm 0.2$ & $150 \pm 50$ & 19 \\
& $(-0.05)$ & $(-0.03)$ & $(-0.92)$ \\
\hline 1643 & $1.6 \pm 0.2$ & $120 \pm 20$ & 19 \\
& $(-0.05)$ & $(-0.06)$ & $(-0.89)$ \\
\hline 2190 & $1.6 \pm 0.2$ & $110 \pm 10$ & 19 \\
& $(-0.05)$ & $(-0.09)$ & $(-0.86)$ \\
\hline
\end{tabular}

In order to better estimate the contribution of time constant $\tau_{2}$, the traces shown in Figure S12A were fit globally such that $\tau_{1}$ (Auger recombination) and $\tau_{3}$ (trapping) were shared, as well as the amplitude of $\tau_{1}$ (given that all five samples should experience the same degree of Auger recombination). We attribute the $\sim 100$ ps component $\tau_{2}$ to static charge transfer with $\mathrm{Me}_{2} \mathrm{BQ}$ molecules preadsorbed to the QD surface, and we do not observe charge recombination as it is likely convoluted with the $20 \mathrm{~ns}$ trapping component discussed earlier. We note that this component comprises, at the highest concentration of $\mathrm{Me}_{2} \mathrm{BQ}, 10 \%$ of the ground state bleach decay, and therefore cannot be primarily responsible for the PL quenching described in the main text given that the same concentration of $\mathrm{Me}_{2} \mathrm{BQ}$ molecule quenches $\sim 95 \%$ of the QDs' PL. 

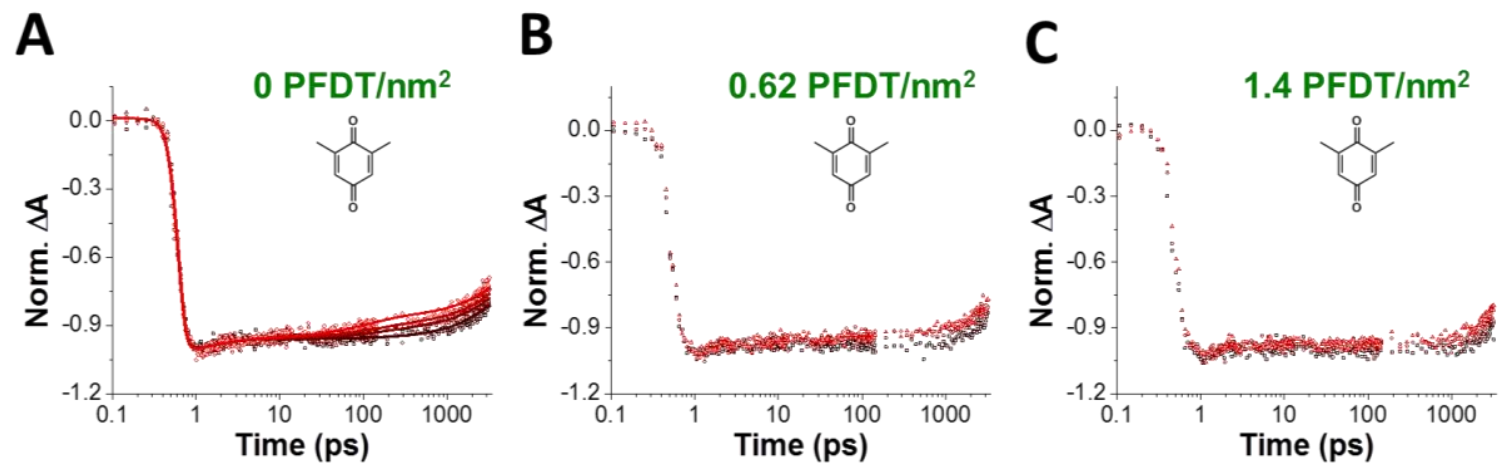

Figure S12. A) Transient kinetics extracted at $983 \mathrm{~nm}$ of PbS QDs capped with native oleate ligands following photoexcitation at $850 \mathrm{~nm}$ after the addition of 0-2190 molar equivalents of $\mathrm{Me}_{2} \mathrm{BQ}$. Solid lines represent fits as described in the text, and indicate that a small portion of photoexcited QDs undergo static charge transfer. Transient kinetic traces of PbS QDs capped with B) 0.62 , or C) $1.4 \mathrm{PFDT} / \mathrm{nm}^{2}$ extracted at $990 \mathrm{~nm}$ and $995 \mathrm{~nm}$ respectively show negligible change in picosecond timescale dynamics before (black) and after the addition of 1095 (maroon), or 2190 (red) molar equivalents of $\mathrm{Me}_{2} \mathrm{BQ}$.

\section{Diffusion-Ordered Spectroscopy (DOSY) NMR. Preparation of Thiol-treated PbS QDs} for DOSY Experiments. We transferred 25 nanomoles $\mathrm{PbS}$ QDs separately from the stock solution (as determined from ground state absorption spectroscopy) into five scintillation vials and removed the hexanes solvent under gentle $\mathrm{N}_{2}$ flow. We brought the QD pellet into a nitrogen glovebox and resuspended each sample in $500 \mu \mathrm{L}$ degassed $\mathrm{d}_{\sigma}$-benzene. We next added $10 \mu \mathrm{L}$ of either 1-decanethiol (DT) or $1 \mathrm{H}, 1 \mathrm{H}, 2 \mathrm{H}, 2 \mathrm{H}$-perfluorodecanethiol (PFDT) to $4 \mathrm{~mL}$ degassed $\mathrm{d}_{6^{-}}$ benzene such that we prepared stock solutions of $12.0 \mathrm{mM}$ DT or $8.7 \mathrm{mM}$ PFDT. We added 49.9 $\mu \mathrm{L}$ (24 eq DT) or $99.8 \mu \mathrm{L}$ (48 eq DT) of decanethiol stock solution to the QDs for the DT/OA capped samples, and we added $86.1 \mu \mathrm{L}$ (30 eq PFDT) or $172.1 \mu \mathrm{L}$ (60 eq PFDT) perfluorodecanethiol stock solution to the QDs for the PFDT/OA samples. A blank sample was prepared with no thiols added. In all cases we next added sufficient solvent to bring the total volume to $760 \mu \mathrm{L}$ (ie such that all solutions were $32.5 \mu \mathrm{M}$ PbS QDs in $\mathrm{d}_{\sigma}$-benzene), and we let the samples sit under $\mathrm{N}_{2}$ for at least $3 \mathrm{~h}$ to equilibrate before we take NMR measurements.

We recorded DOSY spectra of PbS QDs coated with $0,0.50 \mathrm{DT} / \mathrm{nm}^{2}, 1.14 \mathrm{DT} / \mathrm{nm}^{2}, 0.62$ $\mathrm{PFDT} / \mathrm{nm}^{2}, 1.4 \mathrm{PFDT} / \mathrm{nm}^{2}$ as well as spectra of $\mathrm{Me}_{4} \mathrm{BQ}$ and $\mathrm{Me}_{2} \mathrm{BQ}$ in $\mathrm{d}_{6}$-benzene using a double stimulated echo experiment with bipolar gradients (“dstebpgp3s" sequence) on a Bruker Avance-III $600 \mathrm{MHz}$ spectrometer. We used a diffusion delay, $\Delta$, of $0.1 \mathrm{~s}$, a gradient length, $\delta$, of 
$6000 \mu \mathrm{s}$ (PbS QDs) or $2000 \mu \mathrm{s}$ (s-BQs), and chose 16 gradient strength values from 2\% to 80\% (PbS QDs) or 32 gradient strength values from $2 \%$ to $95 \%$ (s-BQs).

In order to determine the diffusion coefficient of these species we plotted the dependence of the total integrated ${ }^{1} \mathrm{H}$ signal of the $\mathrm{PbS} \mathrm{QD}$ samples (originating from bound vinyl oleate protons, $5.69 \mathrm{ppm}$ ), $\mathrm{Me}_{4} \mathrm{BQ}$ (methyl protons, $1.51 \mathrm{ppm}$ ), and $\mathrm{Me}_{2} \mathrm{BQ}$ (quinonoid protons, 6.02 $\mathrm{ppm}$ ) as a function of the gradient function, $G^{2}$, given by eq S4 (Figure S13):

$$
G^{2}=(\gamma g \delta)^{2}\left(\Delta-\frac{\delta}{3}\right)
$$

where $\gamma$ is the gyromagnetic ratio of a proton $\left(2.68 \times 10^{4} \mathrm{~s}^{-1} \mathrm{G}^{-1}\right), g$ is the gradient strength $(\mathrm{G} / \mathrm{cm})$, $\delta$ is the duration of the gradient pulse, and $\Delta$ is the diffusion delay. We performed a fit of the curves in Figure S13 to eq S5,

$$
I\left(G^{2}\right)=I_{0} e^{-D G^{2}}
$$

We calculated the hydrodynamic radii from these diffusion coefficients using the StokesEinstein equation:

$$
r=\frac{k_{B} T}{6 \pi \eta D_{s}}
$$

where $r$ is the hydrodynamic radius, $k_{b}$ is the Boltzmann constant, $T$ is the temperature (295 $\mathrm{K}$ ), and $\eta$ is the viscosity of benzene $(0.59 \mathrm{mPa} \cdot \mathrm{s})$. The results for both $\mathrm{PbS}$ QD and s-BQ samples are found in Table S5.

Table S5. DOSY parameters for s-BQs and PbS QDs.

\begin{tabular}{|l|c|c|}
\hline & $\mathbf{D}\left(\mathbf{c m}^{2} / \mathbf{s}\right)$ & $\mathbf{r}(\mathbf{n m})$ \\
\hline $\mathrm{PbS}$ OA & $(1.10 \pm 0.04) \times 10^{-6}$ & $3.4 \pm 0.1$ \\
\hline $\mathrm{PbS}+30 \mathrm{PFDT}$ & $(1.15 \pm 0.04) \times 10^{-6}$ & $3.2 \pm 0.1$ \\
\hline $\mathrm{PbS}+60 \mathrm{PFDT}$ & $(1.10 \pm 0.06) \times 10^{-6}$ & $3.4 \pm 0.2$ \\
\hline $\mathrm{PbS}+24 \mathrm{DT}$ & $(1.10 \pm 0.04) \times 10^{-6}$ & $3.4 \pm 0.1$ \\
\hline $\mathrm{PbS}+48 \mathrm{DT}$ & $(1.15 \pm 0.04) \times 10^{-6}$ & $3.2 \pm 0.1$ \\
\hline $\mathrm{Me}_{4} \mathrm{BQ}$ & $(1.61 \pm 0.07) \times 10^{-5}$ & $0.23 \pm 0.06$ \\
\hline $\mathrm{Me}_{2} \mathrm{BQ}$ & $(1.84 \pm 0.02) \times 10^{-5}$ & $0.20 \pm 0.02$ \\
\hline
\end{tabular}



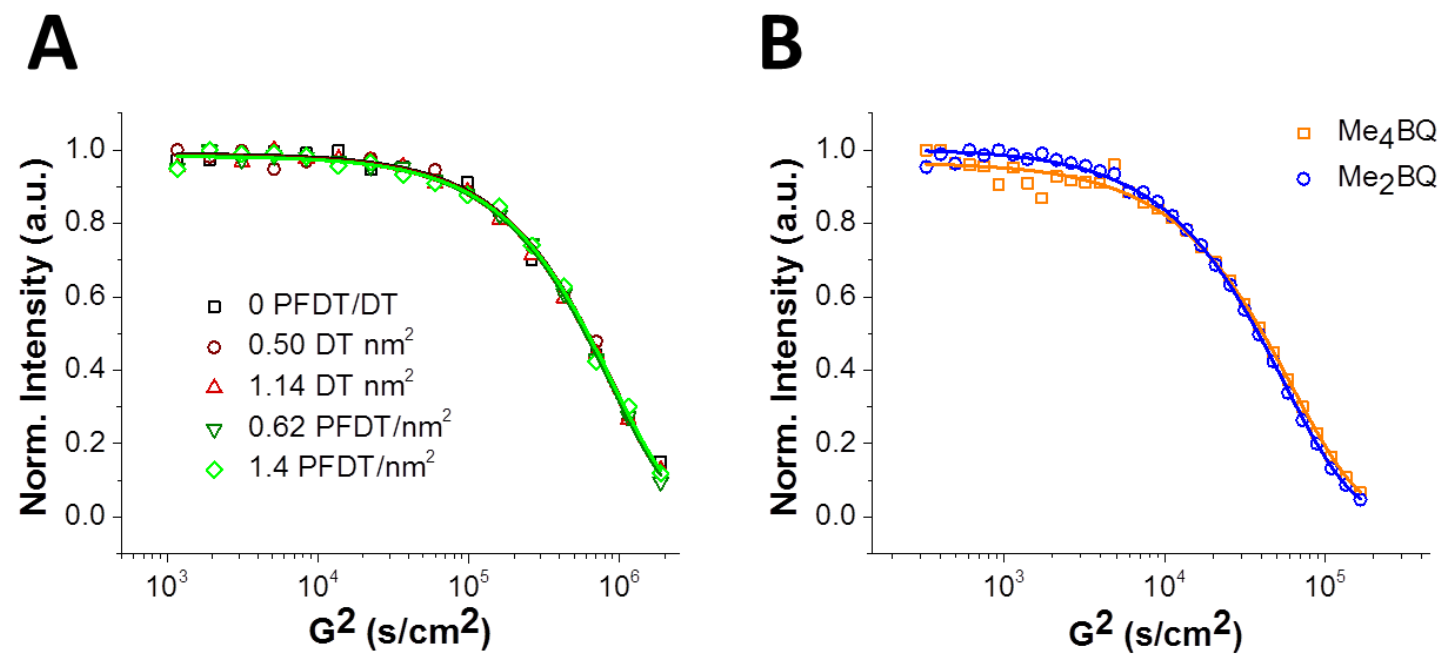

Figure S13. Dependence of signal attenuation on the gradient strength function $\mathrm{G}^{2}$, measured in a DOSY experiment for A) PbS QDs coated with oleate ligands, 0.62 PFDT/nm², 1.4 PFDT/nm², 0.50 DT nm ${ }^{2}$, or $1.14 \mathrm{DT} / \mathrm{nm}^{2}$ and B) $\mathrm{Me}_{2} \mathrm{BQ}$ and $\mathrm{Me}_{4} \mathrm{BQ}$. Solid lines are best fit functions to eq S5 as described in the text.

Constructing Plots of $\Phi / \Phi_{0}$ from Microsecond Transient Absorption Data. In order to verify that the difference in $\Phi / \Phi_{0}$ curves shown in Figure 4 between $\mathrm{Me}_{4} \mathrm{BQ}$ and $\mathrm{Me}_{2} \mathrm{BQ}$ in response to PFDT coverage on the $\mathrm{PbS}$ surface is only due to changes in collisional quenching efficiency, we constructed Stern-Volmer plots from the changes in excitonic lifetime in response to added s-BQ collected in Table S3. Figure S14 contains plots of the ratio of the ground state bleach lifetime of each PFDT/OA mixed monolayer sample with no quencher and the lifetime at each concentration of quencher $\left(\tau_{0} / \tau\right)$. 

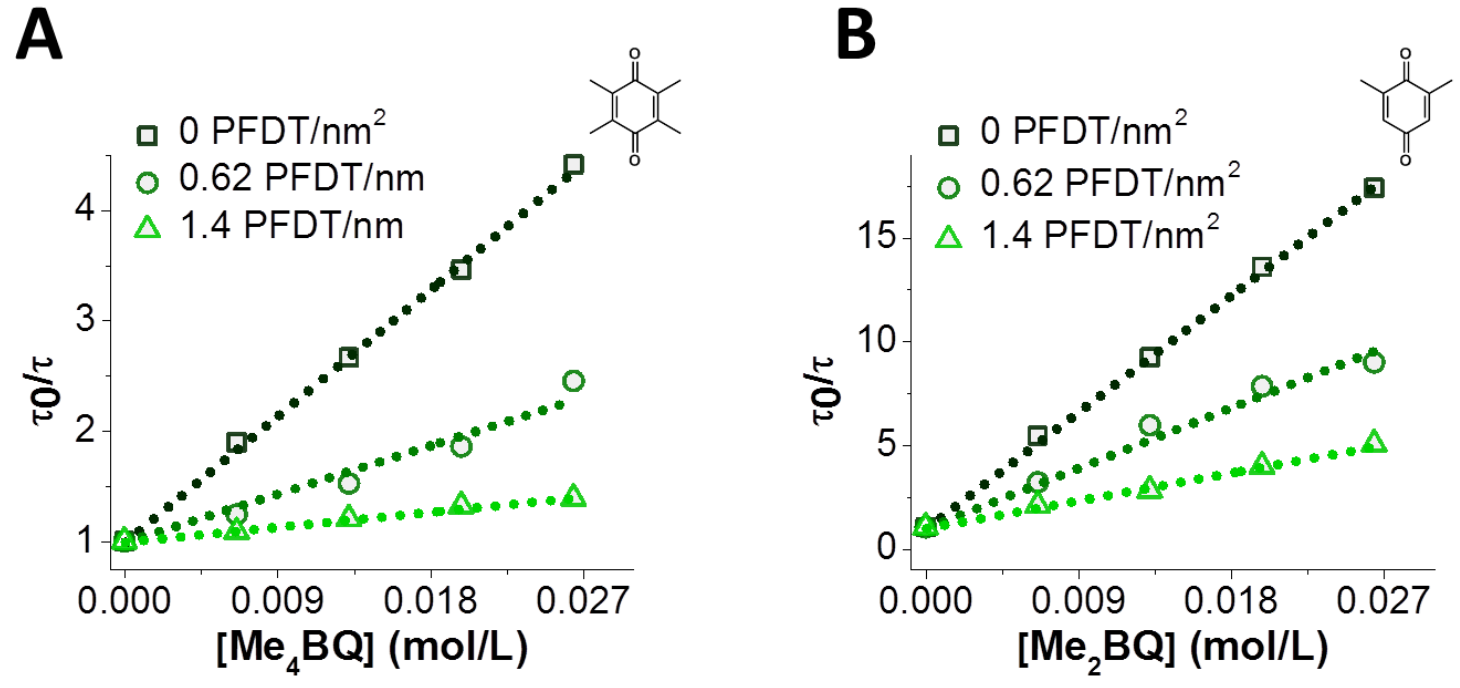

Figure S14. A) Plot of $\tau_{0} / \tau$ (open symbols) for PbS QDs $(11.5 \mu \mathrm{M}$ in benzene) in which the $\mathrm{PbS}$ QDs are coated with OA (black), $0.62 \mathrm{PFDT} / \mathrm{nm}^{2}$ (dark green), or $1.4 \mathrm{PFDT} / \mathrm{nm}^{2}$ (light green) versus the concentration of $\mathrm{Me}_{4} \mathrm{BQ}$ quencher. Colored lines correspond to fits to eq 1 . B) Plot of $\tau_{0} / \tau$ (open symbols) for PbS QDs (11.5 $\mu \mathrm{M}$ in benzene) for the samples described in $(A)$ versus the concentration of $\mathrm{Me}_{2} \mathrm{BQ}$ quencher. All solutions were photoexcited at 850 $\mathrm{nm}$, and transient absorption kinetics were collected $12 \mathrm{~h}$ after addition of 0-2190 equivalents of s-BQ per QD. Colored lines correspond to fits to eq 1.

As in the case of the PL quenching data, all plots of $\tau_{0} / \tau$ are well fit $\left(r^{2}>0.98\right)$ by the Stern Volmer equation (eq 1 in the main text). We use these plots to determine the collisional efficiency $\Phi_{\text {coll }}$ as described in the main text and summarized in Table S6.

Table S6. $\mathrm{Me}_{4} \mathrm{BQ}$ and $\mathrm{Me}_{2} \mathrm{BQ}$ Quenching Parameters for PbS Coated with PFDT/OA Ligand Shells

\begin{tabular}{|c|c|c|c|c|c|c|}
\hline & & & \multicolumn{2}{|c|}{$\mathrm{Me}_{4} \mathrm{BQ}$} & \multicolumn{2}{|c|}{$\mathrm{Me}_{2} \mathrm{BQ}$} \\
\hline \#thiolate/nm ${ }^{2}$ & $\overline{\tau_{\mathbf{0}}(\boldsymbol{\mu} \mathbf{s})^{a}}$ & $\begin{array}{l}k_{0}\left(\mathrm{M}^{-1} \mathrm{~s}^{-1}\right)^{b} \\
\left(\times 10^{10}\right)\end{array}$ & $\begin{array}{l}k_{q}\left(\mathbf{M}^{-1} \mathbf{s}^{-1}\right)^{c} \\
\left(\times 10^{7}\right)\end{array}$ & $\begin{array}{c}\Phi_{\text {coll }}^{d} \\
\left(\times \mathbf{1 0}^{-4}\right)\end{array}$ & $\begin{array}{l}k_{q}\left(\mathrm{M}^{-1} \mathrm{~s}^{-1}\right)^{c} \\
\left(\times 10^{8}\right)\end{array}$ & $\begin{array}{c}\Phi_{\text {coll }}^{d} \\
\left(\times \mathbf{1 0}^{-3}\right)\end{array}$ \\
\hline 0 PFDT/DT & $1.92 \pm 0.02$ & $9.5 \pm 0.3$ & $6.6 \pm 0.1$ & $6.8 \pm 0.2$ & $3.2 \pm 0.4$ & $3.4 \pm 0.1$ \\
\hline 0.62 PFDT & $1.80 \pm 0.03$ & $9.2 \pm 0.3$ & $2.7 \pm 0.1$ & $2.9 \pm 0.2$ & $1.79 \pm 0.08$ & $2.0 \pm 0.1$ \\
\hline 1.4 PFDT & $1.80 \pm 0.02$ & $9.6 \pm 0.5$ & $0.83 \pm 0.02$ & $0.87 \pm 0.05$ & $0.88 \pm 0.03$ & $0.98 \pm 0.05$ \\
\hline
\end{tabular}

${ }^{a}$ Error of monoexponential lifetime fits of PbS GSB.

${ }^{b}$ Error propagated from fitting DOSY measurements.

${ }^{c}$ Error propagated from fitting errors of $\tau_{0}$ and linear fits presented in Figure S14.

${ }^{d}$ Error propagated from $\mathrm{k}_{0}$ and $\mathrm{k}_{\mathrm{q}}$. 


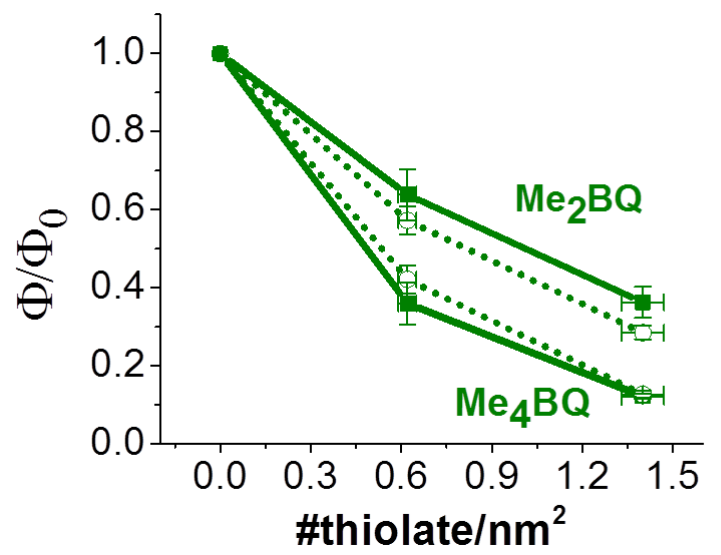

Figure S15. Plot of $\Phi / \Phi_{0}$ for $\mathrm{Me}_{4} \mathrm{BQ}$ and $\mathrm{Me}_{2} \mathrm{BQ}$ for PbS QDs as a function of PFDT thiolate surface coverage as calculated from photoluminescence (solid line, closed symbol) or transient absorption lifetime (dotted line, open symbol) quenching experiments.

We then divide $\Phi_{\text {coll }}$ for PbS QDs coated with PFDT/OA mixed monolayers by $\Phi_{\text {coll }}$ for the $\mathrm{PbS}$ QDs coated exclusively with oleate using the data from Table S6 to construct a plot of $\Phi / \Phi_{0}$, and compare this with the $\Phi / \Phi_{0}$ values calculated from PL quenching experiments. We note that there is good agreement between the collisional quenching efficiency measured for $\mathrm{Me}_{4} \mathrm{BQ}$ by either PL quenching or acceleration of the ground state bleach recovery, and this is reflected by the $\Phi / \Phi_{0}$ curves for $\mathrm{Me}_{4} \mathrm{BQ}$ constructed from these two techniques agreeing within error. The $\Phi / \Phi_{0}$ values for $\mathrm{Me}_{2} \mathrm{BQ}$ measured using microsecond lifetime data are slightly smaller from those measured from photoluminescence quenching. We note that this discrepancy cannot be due to the small amount of static charge transfer observed in systems of OA-capped PbS QDs treated with $\mathrm{Me}_{2} \mathrm{BQ}$, which disappears upon the introduction of PFDT into the ligand shell. We would expect that the elimination of static quenching would decrease, rather than increase the values of $\Phi / \Phi_{0}$ measured through PL experiments (which are sensitive to both static and collisional quenching) relative to those measured through microsecond transient absorption, as the introduction of PFDT would be shutting off electron transfer on both timescales. However, even considering $\Phi / \Phi_{0}$ calculated from microsecond transient absorption, the $\Phi / \Phi_{0}$ curves for $\mathrm{Me}_{2} \mathrm{BQ}$ and $\mathrm{Me}_{4} \mathrm{BQ}$ are different outside the error of the measurement, indicating that the oleophobicity of the PFDT ligand cannot be the only factor determining the permeability of the mixed ligand shell, as described in detail in the main text. 
The agreement between $\Phi / \Phi_{0}$ calculated from PL and microsecond transient absorption results for the PFDT/OA indicate that, for these two s-BQ quencher molecules, monitoring photoluminescence quenching of the PFDT/OA capped QDs is an acceptable way to gauge changes in collisional quenching efficiency as a function of added thiol ligand - that is, the charge transfer with PbS QDs is dominated by microsecond timescale dynamic quenching.

We did not perform the analogous transient absorption experiments for the DT/OA systems, but given (i) our observation that $\mathrm{PbS}$ QDs capped exclusively with oleate do not undergo static quenching with $\mathrm{Me}_{4} \mathrm{BQ}$ and only $10 \%$ of photoexcited QDs undergo static quenching with $\mathrm{Me}_{2} \mathrm{BQ}$, and (ii) our evidence that the DT/OA capped QDs are even less likely to undergo static quenching with these s-BQs than oleate-capped $\mathrm{QDs}^{8}$, we assume that the PL quenching for the DT/OA-capped QDs is also dominated by microsecond timescale dynamic quenching.

\section{References}

(1) Hines, M. A.; Scholes, G. D. Adv. Mater. 2003, 15, 1844.

(2) Moreels, I.; Lambert, K.; Smeets, D.; De Muynck, D.; Nollet, T.; Martins, J. C.; Vanhaecke, F.; Vantomme, A.; Delerue, C.; Allan, G.; Hens, Z. ACS Nano 2009, 3, 3023.

(3) Schneider, C. A.; Rasband, W. S.; Eliceiri, K. W. Nat. Methods 2012, 9, 671.

(4) Amin, V. A.; Aruda, K. O.; Lau, B.; Rasmussen, A. M.; Edme, K.; Weiss, E. A. J. Phys. Chem. C 2015, 119, 19423.

(5) Li, R. F.; Lee, J.; Yang, B. C.; Horspool, D. N.; Aindow, M.; Papadimitrakopoulos, F. J. Am. Chem. Soc. 2005, 127, 2524.

(6) McArthur, E. A.; Morris-Cohen, A. J.; Knowles, K. E.; Weiss, E. A. J. Phys. Chem. B 2010, $114,14514$.

(7) Knowles, K. E.; Malicki, M.; Weiss, E. A. J. Am. Chem. Soc. 2012, 134, 12470.

(8) Malicki, M.; Knowles, K. E.; Weiss, E. A. Chem. Commun. 2013, 49, 4400. 